\title{
Charge fluctuations in a quantum point contact attached to a superconducting lead
}

\author{
Andrew M. Martin, Thomas Gramespacher, and Markus Büttiker \\ Département de Physique Théorique, Université de Genève, CH-1211 Genève 4, Switzerland
}

(Received 19 July 1999)

\begin{abstract}
We show how to calculate the charge noise spectrum in a normal mesoscopic conductor, which is capacitively coupled to a macroscopic gate, when this conductor is attached to $L$ normal leads and $M$ superconducting leads, the only restriction being that the superconducting leads must be at the same chemical potential. We then proceed to examine results for a quantum point contact (QPC) in a normal lead connecting to a superconductor. Of interest is the fluctuating current in a gate capacitively coupled to a QPC. The results are compared with the case when all leads are normal. We find a doubling of the equilibrium charge fluctuations and a large enhancement $(>2)$ in the current noise spectrum to first order in $|\mathrm{eV}|$, when a channel in the QPC is opening. [S0163-1829(99)50342-1]
\end{abstract}

Theory and experimental measurements of the electrical transport properties of mesoscopic conductors, which either contain superconducting regions or are attached to superconducting leads, have generated great interest. Of particular interest are the noise properties of normal superconducting interfaces. While many aspects of the low frequency-current noise spectra ${ }^{1}$ have been understood by generalizing the scattering approach ${ }^{2-6}$ of normal conductors, the fluctuations of the charge have remained unexplored. We generalize the work done for normal mesoscopic conductors ${ }^{7-9}$ to systems which contain superconducting leads. The only restrictions are that there must be one or more normal leads, the superconducting leads must all be at the same chemical potential, and any gates in the structure only see normal regions of the conductor. Having developed this technique, we shall consider one particular example shown in Fig. 1, a quantum point contact (QPC) attached to one normal lead, one superconducting lead and capacitively coupled, via the Coulomb interaction, to a macroscopic gate. We are interested in the charge fluctuations in the hybrid structure which can be measured by observing the current fluctuations at the gate. It turns out that at equilibrium the current fluctuations are determined by the $R C$-time constant. Thus our primary aim is to find the charge relaxation resistance $R$ and the capacitance $C$ for hybrid structures. In the presence of transport, a resistance $R_{V}$, which reflects the shot noise ${ }^{1-3}$ of the hybrid structure, appears.

Consider a mesoscopic conductor with $L$ normal ideal leads and $M$ superconducting leads. The conductor can be described by a scattering matrix with elements $s_{\gamma_{\alpha} \delta_{\beta}}$ which

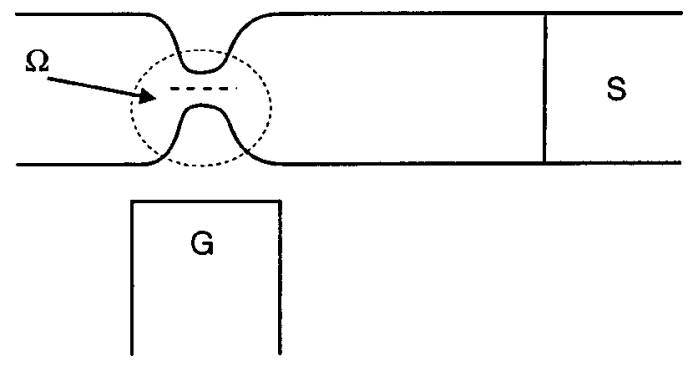

FIG. 1. Quantum point contact, attached to one normal and one superconducting lead, capacitively coupled to a gate. relate particle and hole amplitudes $(\alpha, \beta=p, h)$ incoming at contact $\delta$ to the outgoing amplitudes at contact $\gamma$. Over a region $\Omega$ the mesoscopic conductor is capacitively coupled to a gate. The current fluctuations induced into the gate can be found from the charge fluctuations of the mesoscopic conductor. ${ }^{8,9}$ The investigation of the charge fluctuations starts from the analysis of the bare charge fluctuations which are then screened to find the true charge fluctuations. ${ }^{8,9}$ For a normal conductor the bare charge fluctuations are determined by a density of states matrix which has dimensions $L * L$. For hybrid structure the matrix has dimensions $8 * L * L$ : Any scattering channel, independent of whether the incoming or outgoing channels are electron- or hole-like, can contribute to the electron density at a point $\mathbf{r}$ inside the conductor. Thus to express the density in terms of the scattering matrix requires a conceptual trick: It is useful to imagine that there are two electrostatic potentials, $U^{p}$ and $U^{h}$, which each act separately on the electrons and holes. ${ }^{10}$ The matrix which governs the charge fluctuations can then be found by testing the scattering matrix elements with regard to small variations of the electron and hole potentials, generalizing Refs. 8 and 9. This procedure gives the following density-of-states elements $^{10}$

$$
\begin{aligned}
\mathcal{N}_{\gamma_{\alpha} \delta_{\beta}}^{\eta}\left(\nu_{\lambda}, \mathbf{r}\right)= & \frac{-1}{4 \pi i}\left[s_{\nu_{\lambda} \gamma_{\alpha}}^{\dagger}[E, \underline{U}(\mathbf{r})] \frac{\partial s_{\nu_{\lambda} \delta_{\beta}}[E, \underline{U}(\mathbf{r})]}{q^{\eta} \partial U^{\eta}(\mathbf{r})}\right. \\
& \left.-s_{\nu_{\lambda} \gamma_{\alpha}}[E, \underline{U}(\mathbf{r})] \frac{\partial s_{\nu_{\lambda} \delta_{\beta}}^{\dagger}[E, \underline{U}(\mathbf{r})]}{q^{\eta} \partial U^{\eta}(\mathbf{r})}\right]
\end{aligned}
$$

where the labels $\nu, \gamma$, and $\delta$ denote contacts $(1 \ldots L), \alpha, \beta$, $\lambda$, and $\eta$ denote the electron/hole degrees of freedom $(p / h)$ and $q^{p}=e=-q^{h}$. The functional derivatives are taken at the equilibrium electrostatic potential $U^{p}=U^{h}=U_{e q}$. To give an example, $\mathcal{N}_{1 p 2 p}^{h}\left(1_{h}, \mathbf{r}\right)$ is the hole density associated with two electron current amplitudes incident from contacts 1 and 2 and a reflected outgoing hole amplitude in contact 1 . With the help of these basic expressions we can now find both the average density of states as well as the fluctuations. The hole density of states of a region $\Omega$ of the conductor is $N^{h}$ $=N^{h}(p)+N^{h}(h)$, where 


$$
N^{h}(\alpha)=\sum_{\nu \gamma \lambda} \int_{\Omega} d^{3} \mathbf{r} \operatorname{Tr}\left[\mathcal{N}_{\gamma_{\alpha} \gamma_{\alpha}}^{h}\left(\nu_{\lambda}, \mathbf{r}\right)\right]
$$

and the particle density of states $N^{p}=N^{p}(p)+N^{p}(h) \quad$ with

$$
N^{p}(\alpha)=\sum_{\nu \gamma \lambda} \int_{\Omega} d^{3} \mathbf{r} \operatorname{Tr}\left[\mathcal{N}_{\gamma_{\alpha} \gamma_{\alpha}}^{p}\left(\nu_{\lambda}, \mathbf{r}\right)\right] .
$$

The trace is over open quantum channels. $N^{\alpha}(\beta)$ is the injectivity of particles (holes) $[\beta=p(h)]$, from all contacts into the conductor, given a change in the particle (hole) potential $[\alpha=p(h)]$.

The fluctuations of the bare charge in a region $\Omega$ can be found from the charge operator $e \hat{\mathcal{N}}$ given by

$$
\begin{aligned}
e \hat{\mathcal{N}}(\omega)= & \sum_{\substack{\gamma \alpha \\
\delta \beta}} \sum_{\eta \nu \lambda} \int_{\Omega} d^{3} \mathbf{r} \int d E \hat{a}_{\gamma_{\alpha}}^{\dagger}(E) q^{\eta} \\
& \times \mathcal{N} \underset{\gamma_{\alpha} \delta_{\beta}}{\eta}\left(\nu_{\lambda}, \mathbf{r} ; E, E+\hbar \omega\right) \hat{a}_{\delta_{\beta}}(E+\hbar \omega),
\end{aligned}
$$

where the zero-frequency limit of $\mathcal{N}_{\gamma_{\alpha} \delta_{\beta}}^{\eta}\left(\nu_{\lambda}, \mathbf{r} ; E, E+\hbar \omega\right)$ is given by Eq. (1). In Eq. (4), $\hat{a}_{\gamma \alpha}^{\dagger}(E)$ creates an incoming electron/hole $(\alpha=p / h)$ in lead $\gamma$. The true charge fluctuations must be obtained by taking into account the Coulomb interaction and below we show how to obtain the true charge fluctuations from the fluctuations of the bare charges.

Given Eqs. (1)-(4) our next step is to consider the system shown in Fig. 1 and to calculate the charge fluctuations in the mesoscopic region and hence deduce the current fluctuations in the gate. To do this it helps to first consider an approach which allows us to correctly calculate the charge $\delta Q$ in the mesoscopic region when a voltage is applied across the system. The charge accumulated underneath the gate in the region $\Omega$ can be expressed in two ways: $:^{7-9}$ If we describe the Coulomb interaction with the help of a geometrical capacitance $C$ the incremental charge is simply related to the potential variations in the conductor $\delta U$ and the gate $\delta V_{g}$ via $\delta Q=C\left(\delta U-\delta V_{g}\right)$, assuming that the gate is macroscopic. On the other hand, the charge $\delta Q$ is also the sum of the injected charges due to the variation of the contact potentials (keeping the internal electrostatic potential fixed) and the induced charges which are generated by the Coulomb interaction. Thus

$$
\begin{aligned}
\delta Q= & C\left(\delta U-\delta V_{g}\right) \\
= & \frac{1}{2}\left[q^{p} N^{p}(p) \delta \mu_{p}+q^{h} N^{h}(p) \delta \mu_{p}+q^{h} N^{h}(h) \delta \mu_{h}\right. \\
& +q^{p} N^{p}(h) \delta \mu_{h}-q^{p} N^{p}(p) q^{p} \delta U_{p}-q^{p} N^{p}(h) q^{h} \delta U_{h} \\
& \left.-q^{h} N^{h}(p) q^{p} \delta U_{p}-q^{h} N^{h}(h) q^{h} \delta U_{h}\right] .
\end{aligned}
$$

The first four terms in Eq. (5) determine the charge injected into the conductor as a consequence of the variation of the contact potentials. Similarly to the conceptual separation of the electrostatic potential, we have here assumed that each contact has separate electrochemical potentials $\mu_{p}$ and $\mu_{h}$ for electrons and holes. ${ }^{4}$ In reality $\mu_{p}=-\mu_{h}=\mu=\mathrm{eV}$. The second four terms in Eq. (5) determine the induced charges. In the absence of superconductivity Eq. (5) reduces to the "Poisson equation" for charge variations in a normal conductor: ${ }^{7,9}$ We have $N^{p}(h)=N^{h}(p)=0$ and $N \equiv N^{p}(p)$ $=N^{h}(h)$. Going back to Eq. (5) we can solve it for $\delta U$ and can use this solution to determine the electrochemical capacitance of the hybrid structure vis-à-vis the gate. We find

$$
C_{\mu}^{N S} \equiv \frac{\delta Q}{\delta V} \equiv-\frac{\delta Q}{\delta V_{g}}=\frac{C e^{2} N_{\Sigma}}{C+e^{2} N_{\Sigma}}
$$

with a total density of states

$$
N_{\Sigma}=\frac{1}{2}\left[N^{p}(p)-N^{p}(h)+N^{h}(h)-N^{h}(p)\right] .
$$

It is instructive to examine $C_{\mu}^{N S}$ for an ideal ballistic wire. For a perfect $N-S$ structure, $C_{\mu}$ is zero, since every charge incident upon the conductor is perfectly Andreev reflected and hence the net accumulated charge is zero. If the superconductor is driven into the normal state, the capacitance $e^{7,11}$ is $C_{\mu}^{N}=C e^{2} N /\left(C+e^{2} N\right)$ which for a ballistic wire reduces to $C_{\mu}^{N}=C$ since typically $e^{2} / C \gg 1 / N$. Thus $C_{\mu}^{N S}$ for the hybrid structure can differ dramatically from $C_{\mu}^{N}$.

If we now wish to consider fluctuations in the charge then we have to consider the Poisson equation for the fluctuating charges. ${ }^{9,8}$ For the case that the voltages are held fixed (zeroimpedance external circuit) this leads to an operator equation,

$$
\hat{Q}=C \hat{U}=e \hat{\mathcal{N}}-e^{2} N_{\Sigma} \hat{U}
$$

where $\hat{\mathcal{N}}$ is the operator of the bare charge fluctuations given by Eq. (4) and the last term in Eq. (8) describes the screening of the bare charge fluctuations. Solving Eq. (8) for $\hat{U}$ we can express the charge fluctuations $\hat{Q}$ in terms of the bare charge fluctuations. ${ }^{9}$ For the fluctuation spectrum of the charge this gives

$$
\begin{aligned}
S_{Q Q}(\omega)= & (1 / 2) C_{\mu}^{2} N_{\Sigma}^{-2} \sum_{\gamma \delta} \sum_{\alpha \beta} \int d E F_{\gamma_{\alpha} \delta_{\beta}}(E, \hbar \omega) \\
& \times \operatorname{Tr}\left[\mathcal{N}_{\gamma_{\alpha} \delta_{\beta}}(E, E+\hbar \omega) \mathcal{N}_{\gamma_{\alpha} \delta_{\beta}}^{\dagger}(E, E+\hbar \omega)\right],
\end{aligned}
$$

where

$$
\mathcal{N}_{\gamma_{\alpha} \delta_{\beta}}\left(E, E^{\prime}\right)=\sum_{\eta \nu \lambda} \operatorname{sgn}\left(q^{\eta}\right) \int_{\Omega} d^{3} \mathbf{r} \mathcal{N}_{\gamma_{\alpha} \delta_{\beta}}^{\eta}\left(\nu_{\lambda}, \mathbf{r} ; E, E^{\prime}\right),
$$

$$
F_{\gamma_{\alpha} \delta_{\beta}}\left(E, E^{\prime}\right)=f_{\gamma_{\alpha}}(E)\left[1-f_{\delta_{\beta}}\left(E^{\prime}\right)\right]+f_{\delta_{\beta}}\left(E^{\prime}\right)\left[1-f_{\gamma_{\alpha}}(E)\right]
$$

In the above the sum is over all normal contacts $\gamma \delta$ and degrees of freedom $\alpha \beta$. The Fermi functions $f_{\gamma \alpha}(E)$ are defined such that $f_{\gamma_{p}}=f_{0}\left(E_{p}-\mu_{\gamma}\right)$ and $f_{\gamma_{h}}=f_{0}\left(E_{h}+\mu_{\gamma}\right)$, where $E_{\alpha}$ is the energy of a particle (hole) $[\alpha=p(h)]$ in reservoir $\gamma$, which is at a chemical potential $\mu_{\gamma}, f_{0}(E)$ is the Fermi function at the condensate chemical potential of the superconducting leads $\left(\mu_{0}\right)$.

Now we evaluate Eq. (9) at equilibrium and zero temperature, to leading order in $\hbar \omega \ll|\Delta|$. We find

$$
S_{Q Q}(\omega)=2 C_{\mu}^{2} R_{q} \hbar|\omega|
$$

with a charge relaxation resistance 


$$
R_{q}=\frac{h}{4 e^{2}} \frac{\sum_{\gamma \delta} \Sigma_{\alpha \beta} \operatorname{Tr}\left(\mathcal{N}_{\gamma_{\alpha} \delta_{\beta}} \mathcal{N}_{\gamma_{\alpha} \delta_{\beta}}^{\dagger}\right)}{\left[N_{\Sigma}\right]^{2}} .
$$

In the zero frequency limit, and at a temperature $k T \ll|\Delta|$ the charge fluctuations are given by $S_{Q Q}(\omega)=2 C_{\mu}^{2} R_{q} k T$ with $R_{q}$ as given by Eq. (13). If we bring the hybrid structure into a nonequilibrium state by applying a bias $e|V| \gg k T$ between the normal reservoir and the superconductor, we find at zero temperature in the low-frequency limit to leading order in $e|V|$ for the system shown in Fig. 1,

$$
S_{Q Q}(\omega)=2 C_{\mu}^{2} R_{V} e|V|
$$

with a nonequilibrium resistance

$$
R_{V}=\frac{h}{4 e^{2}} \frac{\operatorname{Tr}\left(\mathcal{N}_{1_{p}{ }_{h}{ }} \mathcal{N}_{1_{p} 1_{h}}^{\dagger}+\mathcal{N}_{1_{h} 1_{p}} \mathcal{N}_{1_{h} 1_{p}}^{\dagger}\right)}{\left[N_{\Sigma}\right]^{2}} .
$$

The resistance $R_{V}$ is a consequence of the charge fluctuations which arise due to the shot noise of the transport state. The fluctuation spectra of the current at the gate $G$ in Fig. (1) are related to the charge fluctuation spectra Eqs. (12) and (14) via $S_{I I}(\omega)=\omega^{2} S_{Q Q}(\omega)$.

To proceed further we follow Beenakker ${ }^{5}$ and express the electron-hole scattering matrix of the $N-S$ structure in terms of the scattering matrix elements of the normal structure. For simplicity we restrict our considerations here to normal structures which are symmetric with respect to left and right going carriers. The scattering matrix of the normal conductor is then given by a reflection matrix $r$ and a transmission matrix $t$ only. In terms of $r$ and $t$ the electron-hole scattering matrix elements are

$$
\begin{gathered}
s_{1_{p} 1_{p}}(E)=r_{p}(E)+\alpha^{2} t_{p}(E) r_{h}(-E) M_{p}(E) t_{p}(E) \\
s_{1_{h} 1_{p}}(E)=\alpha e^{-i \phi} t_{h}(-E) M_{p}(E) t_{p}(E) \\
s_{1_{h}{ }_{h} h}(E)=r_{h}(E)+\alpha^{2} t_{h}(E) r_{p}(-E) M_{h}(E) t_{h}(-E) \\
s_{1_{p} 1_{h}}(E)=\alpha e^{i \phi} t_{h}(E) M_{h}(E) t_{p}(-E)
\end{gathered}
$$

where $\alpha=\exp [-i \arccos (E / \Delta)]$ and

$$
\begin{aligned}
& M_{p}(E)=\left[1-\alpha^{2} r_{p}(E) r_{h}(-E)\right]^{-1}, \\
& M_{h}(E)=\left[1-\alpha^{2} r_{h}(E) r_{p}(-E)\right]^{-1} .
\end{aligned}
$$

In Eqs. (16)-(21) we have given an index $p, h$ to $r$ and $t$ to indicate whether a carrier or a hole is scattered since we have to distinguish derivatives with respect to the electron and hole potentials. Throughout the rest of the work presented here we will be making use of particle-hole symmetry (i.e., $\left.E \rightarrow 0, s_{p}=s_{h}^{*}\right)$.

Now we wish to calculate the properties of the system shown in Fig. 1. We choose to model a quantum point contact, using a saddle point potential ${ }^{12} V(x, y)=V_{0}+\frac{1}{2} m \omega_{y}^{2} y^{2}$ $-\frac{1}{2} m \omega_{x}^{2} x^{2}$, and as in Ref. 9 we have evaluated the density of states semi-classically. We also take the Wentzel-KramersBrillouin (WKB) limit allowing us to make the following transformation: ${ }^{8}-\int_{\Omega} d^{3} \mathbf{r}\left[\partial / \partial q^{\lambda} U^{\lambda}(r)\right] \rightarrow d / d E^{\lambda}$. In the above transformation we have been careful to keep the index

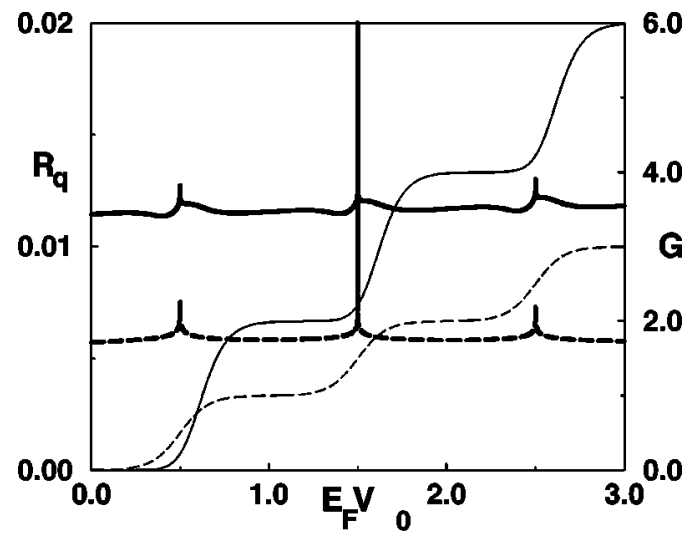

FIG. 2. $R_{q}^{N S}$ (thick solid line) and $R_{q}^{N}$ (thick dashed line), in units of $h / e^{2} . G^{N S}$ (thin solid line) and $G^{N}$ (thin dashed line) is the conductance, in units of $e^{2} / h$, as a function of $E_{F}-V_{0}$ in units of $\hbar \omega_{y}$, for a saddle QPC with $\omega_{y} / \omega_{x}=2$.

$\lambda$ included, such that we can distinguish between particles and holes. We also note that we can write $d s / d E^{\lambda}$ $=\left(d s / d \theta^{\lambda}\right)\left(d \theta^{\lambda} / d E^{\lambda}\right)$, where $d \theta^{\lambda} / d E^{\lambda}$ is the particle (hole) $[\lambda=p(h)]$ density of states divided by $\pi$ in the conductor in region $\Omega$. Making use of particle-hole symmetry we have $d \theta / d E=d \theta^{p} / d E^{p}=d \theta^{h} / d E^{h}$.

Using Eqs. (13), (16)-(19) we now calculate $R_{q}$ and find

$R_{q}^{N S}=\frac{h}{2 e^{2}} \frac{\sum_{n}\left[16 R_{n}^{6}+R_{n}\left(4 R_{n}+1\right)^{2} T_{n}^{2}\right] /\left(1+R_{n}\right)^{4}\left(\frac{d \theta_{n}}{d E}\right)^{2}}{\left[\sum_{n} \frac{4 R_{n}^{3}}{\left(1+R_{n}\right)^{2}}\left(\frac{d \theta_{n}}{d E}\right)\right]^{2}}$ as compared $^{9,13}$ to

$$
R_{q}^{N}=\frac{h}{4 e^{2}} \frac{\sum_{n}\left(\frac{d \theta_{n}}{d E}\right)^{2}}{\left[\sum_{n}\left(\frac{d \theta_{n}}{d E}\right)\right]^{2}}
$$

for a QPC attached to two normal leads, where $T_{n}$ and $R_{n}$ $=1-T_{n}$ are the transmission and reflection probabilities of the $n$th quantum channel and $\theta_{n}$ is the phase accumulated by a carrier in the $n$th channel during transmission through the QPC. In Fig. 2 we plot $R_{q}^{N S}$ and $R_{q}^{N}$ (the charge relaxation resistance for a QPC attached to two normal leads). For the parameters we have chosen, we see that the difference between $R_{q}^{N}$ and $R_{q}^{N S}$ is roughly a factor of 2. This arises from the difference between the contact resistance of a mesoscopic conductor in an $N-S$ system compared to an $N-N$ system. ${ }^{14}$

Having examined $R_{q}^{N S}$ and $R_{q}^{N}$ we now proceed to examine $R_{V}^{N S}$, finding

$$
R_{V}^{N S}=\frac{h}{2 e^{2}} \frac{\sum_{n} \frac{R_{n}\left(4 R_{n}+1\right)^{2} T_{n}^{2}}{\left(1+R_{n}\right)^{4}}\left(\frac{d \theta_{n}}{d E}\right)^{2}}{\left[\sum_{n} \frac{4 R_{n}^{3}}{\left(1+R_{n}\right)^{2}}\left(\frac{d \theta_{n}}{d E}\right)\right]^{2}}
$$




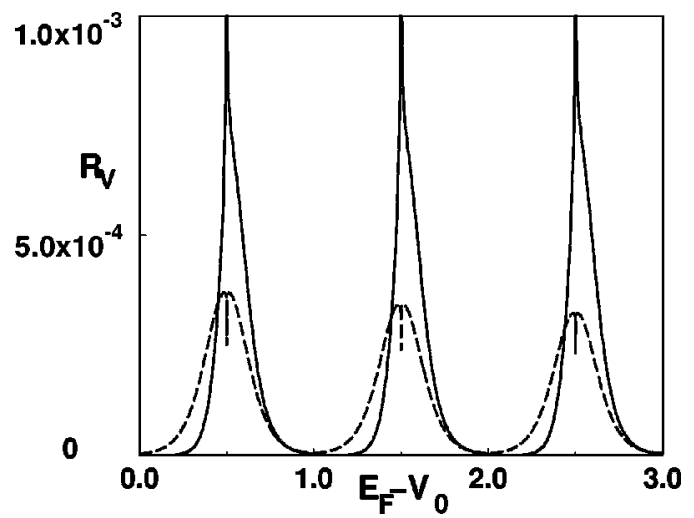

FIG. 3. $R_{V}^{N S}$ (solid line) and $R_{V}^{N}$ (dashed line), in units of $h / e^{2}$, as a function of $E_{F}-V_{0}$ in units of $\hbar \omega_{y}$, for a saddle QPC with $\omega_{y} / \omega_{x}=2$.

as compared ${ }^{9}$ to

$$
R_{V}^{N}=\frac{h}{e^{2}} \frac{\sum_{n} \frac{1}{4 R_{n} T_{n}}\left(\frac{d T_{n}}{d E}\right)^{2}}{\left[\sum_{n}\left(\frac{d \theta_{n}}{d E}\right)\right]^{2}}
$$

for a QPC attached to two normal leads. In Fig. 3 we see both $R_{V}^{N S}$ and $R_{V}^{N}$ as a function of $E_{F}-V_{0}$. At the channel threshold $R_{n}=T_{n}=1 / 2$ the nonequilibrium resistance $R_{V}^{N S}$ of the hybrid structure tends to $(9 / 4)\left(h / e^{2}\right)$ whereas the normal state $R_{V}^{N S}$ is completely suppressed at the channel threshold due to the divergence of the semiclassical density of states.

In this work we have taken the first steps in considering frequency-dependent charge fluctuations and noise calculations in mesoscopic normal superconducting hybrid systems. To do this we have considered structures where the condensate chemical potentials of any superconducting lead remains the same and gates only see the normal regions of the conductor. This allows us to consider oscillating voltages in the normal reservoirs and calculate the systems response. We have focused on the $R C$ time of such structures. The $R C$ time is a fundamental dynamical quantity of electrical conductors. Elsewhere we have shown that for normal mesoscopic conductors the $R C$ time also determines the dephasing rate of Coulomb coupled structures. ${ }^{15}$ We expect that such a relation also holds for hybrid systems. As an example we considered the most simple nontrivial example, a QPC attached to one normal lead and one superconducting lead. We have given a firm prediction that the $R C$ time and the fluctuations induced into a gate differ markedly for an $N-S$ structure and a normal conductor. As usual, we assume perfect Andreev reflection at the superconducting interface, whereas at real interfaces only partial Andreev reflection might occur. Currents induced into gates can be measured. ${ }^{16}$ We believe that it is similarly possible to measure the current fluctuations at a gate.

This work was supported by the Swiss National Science Foundation and by the TMR network Dynamics of Nanostructures.
${ }^{1}$ V. A. Khlus, Zh. Éksp. Teor. Fiz. 93, 2179 (1987) [Sov. Phys. JETP 66, 1243 (1987)].

${ }^{2}$ M. J. M. de Jong and C. W. J. Beenakker, Phys. Rev. B 49, 16070 (1994).

${ }^{3}$ B. A. Muzykantskii and D. E. Khmelnitskii, Phys. Rev. B 50, 3982 (1994).

${ }^{4}$ M. P. Anantram and S. Datta, Phys. Rev. B 53, 16390 (1996); S. Datta, P. F. Bagwell, and M. P. Anantram, Phys. Low-Dimens. Semicond. Struct. 3, 1 (1996).

${ }^{5}$ C. W. J. Beenakker, Rev. Mod. Phys. 69, 731 (1997).

${ }^{6}$ For reviews, see Superlattices Microstruct. 25, 627-1288 (1999) (special editor P. F. Bagwell).

${ }^{7}$ M. Büttiker, H. Thomas, and A. Pretre, Phys. Lett. A 180, 364 (1993).

${ }^{8}$ M. Büttiker, J. Math. Phys. 37, 4793 (1996).

${ }^{9}$ M. H. Pedersen, S. A. van Langen, and M. Büttiker, Phys. Rev. B
57, 1838 (1998).

${ }^{10} \mathrm{~T}$. Gramespacher and M. Büttiker, cond-mat/9908469 (unpublished).

${ }^{11}$ B. Wang, X. Zhao, J. Wang, H. Guo, Appl. Phys. Lett. 74, 2887 (1999); H. Wei, N. Zhu, J. Wang, and H. Guo, Phys. Rev. B 56, 9657 (1997).

${ }^{12}$ M. Büttiker, Phys. Rev. B 41, 7906 (1990).

${ }^{13}$ The factor $1 / 4$ was mistakenly omitted in $R_{q}$ as given in Ref. 9 and is herewith corrected.

${ }^{14}$ F. Sols and J. Sánchez-Cañizares, Superlattices Microstruct. 25, 627 (1999).

${ }^{15}$ M. Büttiker and A. M. Martin, cond-mat/9902320 (unpublished).

${ }^{16}$ W. Chen, T. P. Smith III, M. Büttiker, and M. Shayegan, Phys. Rev. Lett. 73, 146 (1994); P. K. H. Sommerfeld, R. W. van der Heijden, and F. Peeters, Phys. Rev. B 53, R13 250 (1996). 